\title{
Immunological signature of amyopathic dermatomyositis
}

\section{PARIS}

寥DIDEROT

Inserm

\section{Proof of systemic inflammation}

C.CASSIUS ${ }^{12}$, R.AMODE ${ }^{12}$, M.JACHIET ${ }^{1}$, M.BRANCHTEIN 2, M.BAGOT ${ }^{12}$

A.BENSSUSAN ${ }^{2}$, M.BATTISTELLA ${ }^{3}$, H.LE BUANEC ${ }^{2 *}$, JD.BOUAZIZ $^{1}{ }^{2}$ *

${ }^{1}$ Dermatology Department, ${ }^{2}$ INSERM U976 and ${ }^{3}$ Pathology Department, Saint-

Louis hospital, Assistance Publique-Hôpitaux de Paris, Paris, France. *co-last
LARIBOISIÈRE

FERNAND-WIDAL

Institut national
de la santé et de

1

\section{Why studying aDM?}

Dermatomyositis (DM) is an auto-immune mediated inflammatory myopathy associated with specific skin symptoms.

Amyopathic DM (aDM) is characterized by the lack of muscle involvement (classical DM (cDM) = skin + muscle).

No specific immunological investigation has been performed to date in aDM patients.

\section{How studying aDM?}

8 aDM patients (before any suppressive treatment), 19 healthy blood donors (for blood mononuclear cells and serum) and 8 normal skin biopsies (control for skin transcriptomic study) were analyzed.

T cell population phenotyping was performed using mutiparameter flow cytometry ( 18 colors).

Cytokine level in the serum was measured using Luminex technology.

Total RNA was extracted from snap frozen skin sections for hybridization on Human Transcriptome Array 2,0 chip (Affymetrix). Microarray data were analyzed with the Linear Model for Microarray data suite for « $R$ » (The R foundation).

Lymphocytes immunological signature

DM

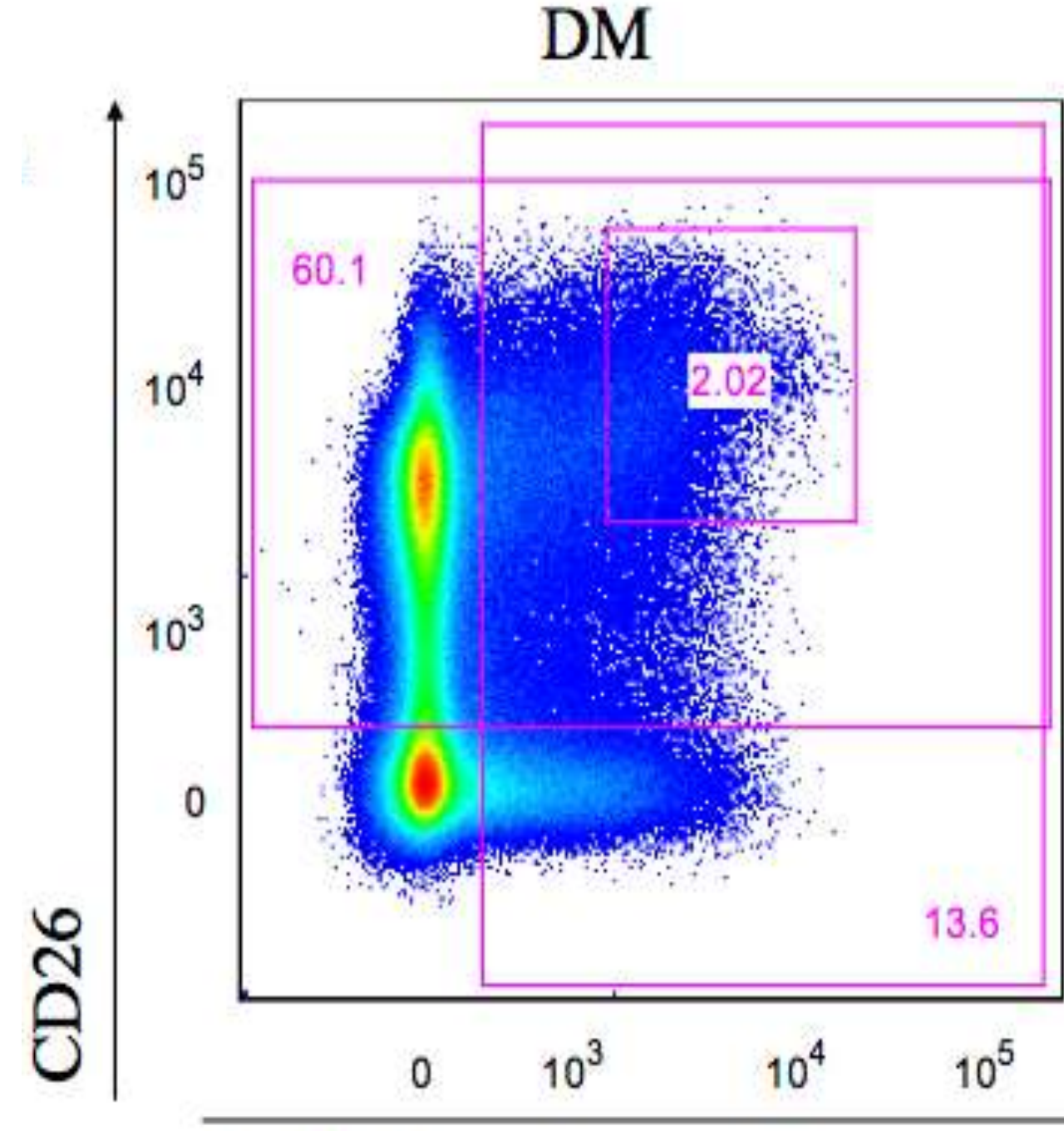

CD161

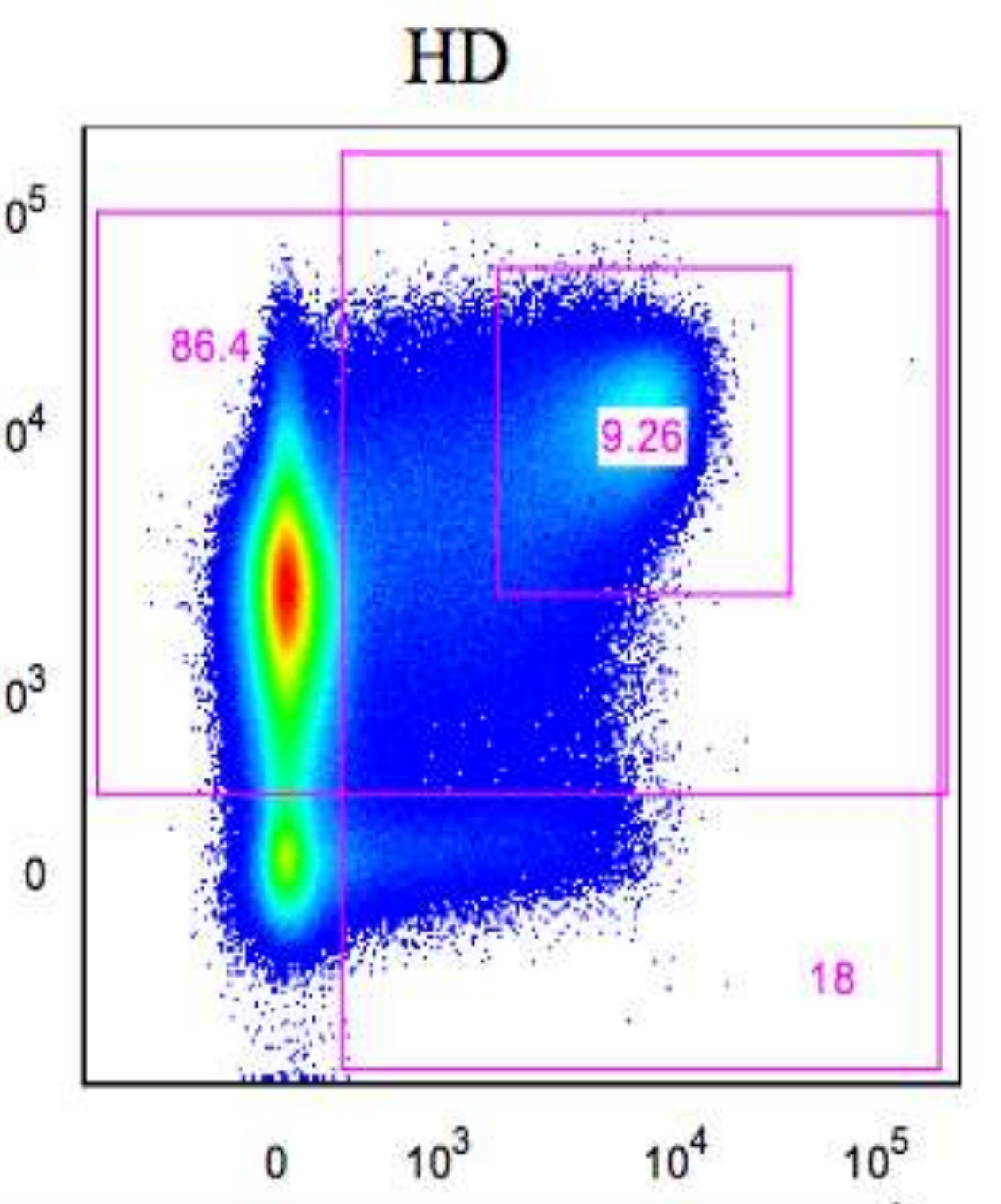

ใิ|

$$
\mathrm{CD} 4
$$

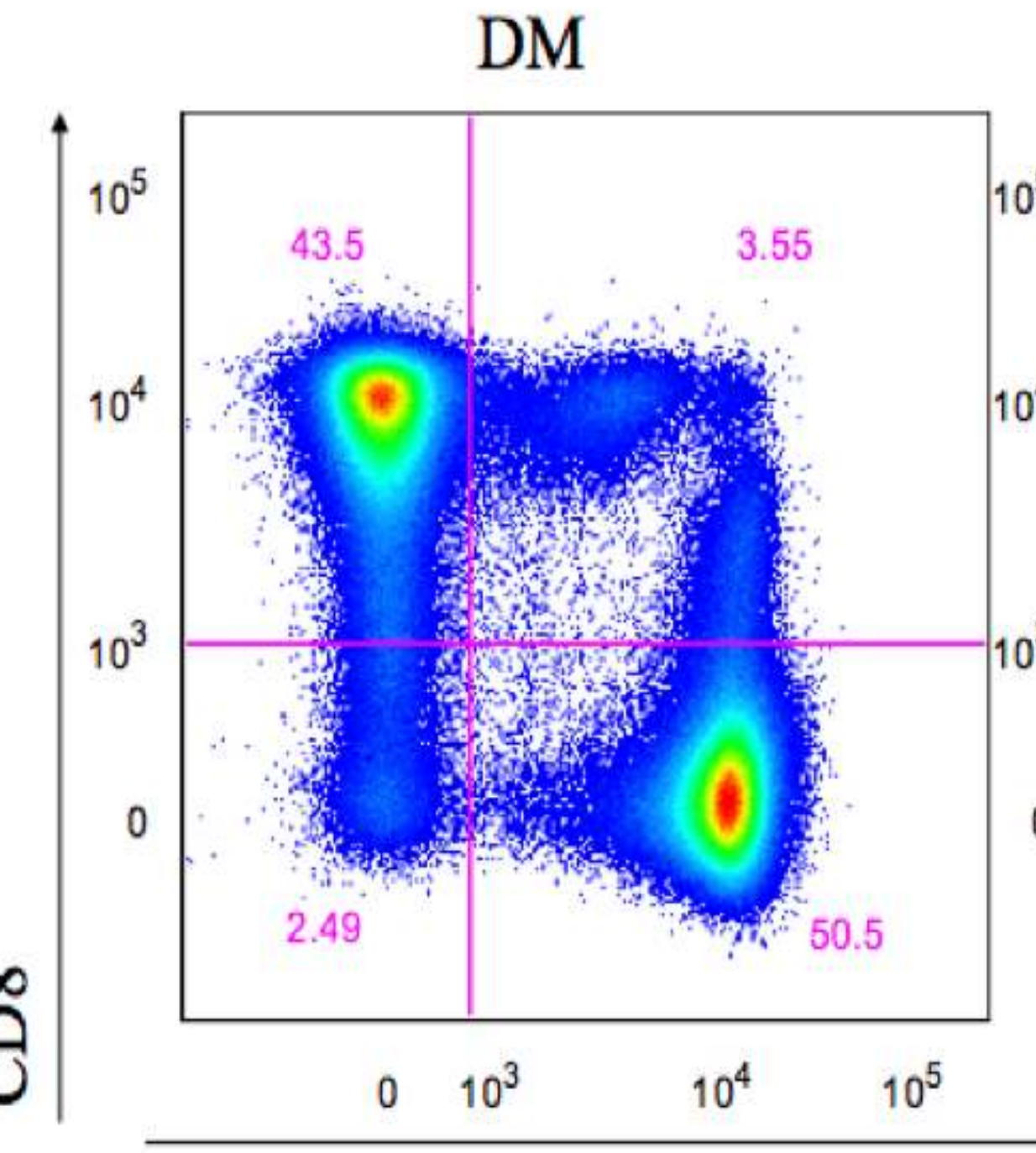

a. Decreased frequency of CD3+CD26+CD161+ (identifying MAIT cells)

The CD3+CD26+CD161+ subpopulation median frequency was 8,45 in amyopathic dermatomyositis patients $(n=7)$ and 13,7 in healthy donors $(n=19)$. $p<0,01$ b. Decreased frequency of CD3+CD4-CD8- (known as regulatory population)

The median percentage of CD3+CD4-CD8- $T$ cells was 0,791 in amyopathic dermatomyositis $(n=7)$ and $1,49(\mathrm{n}=19)$ in healthy donors. $p<0,05$

$\mathrm{HD}$

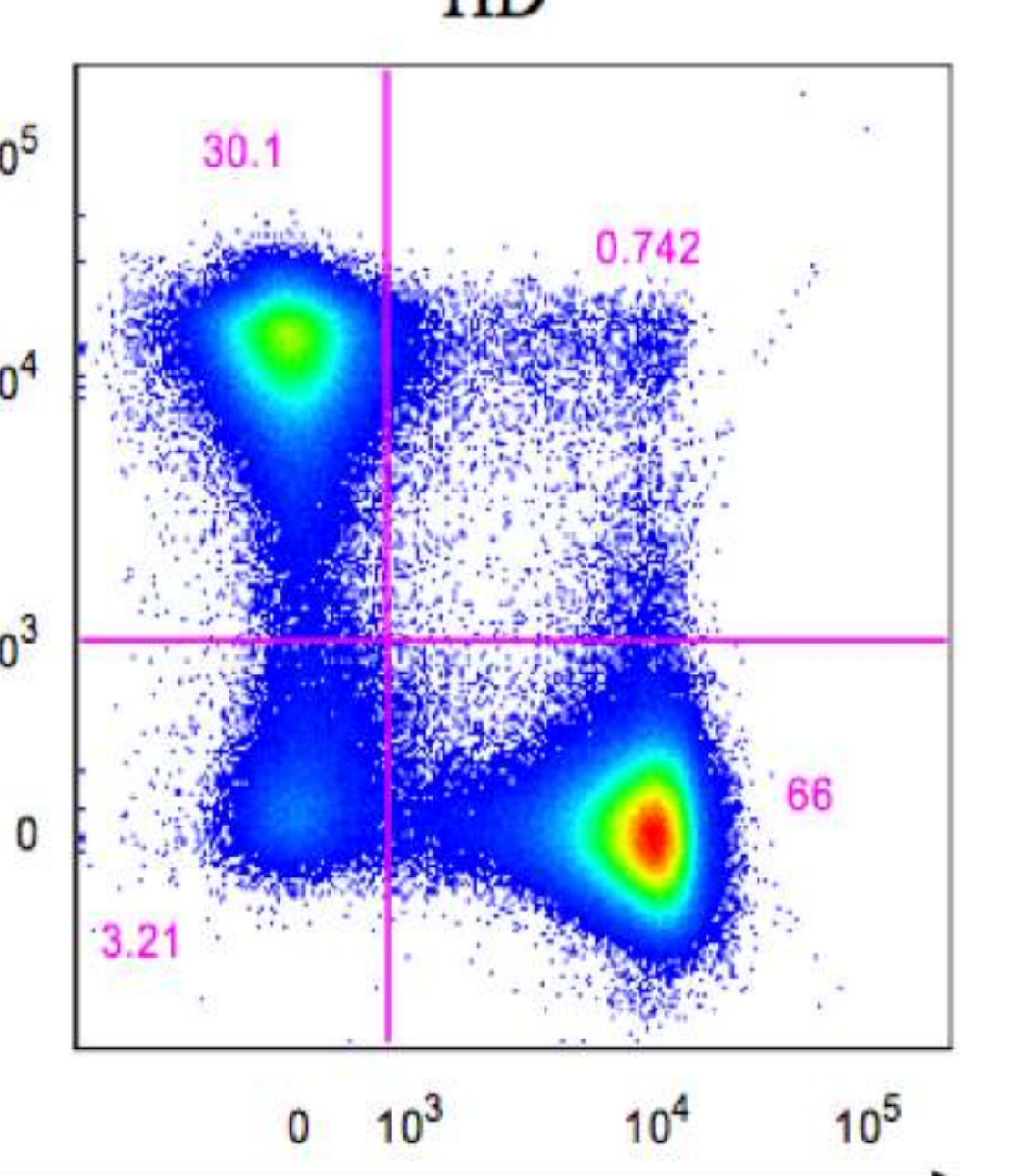

ปิ่|$$
\text { ปे }
$$

\section{CD127}

Conserved frequency of Tregs characterized by $\mathrm{CD} 3+\mathrm{CD} 4+\mathrm{CD} 25+$ and $\mathrm{CD} 127-$ and by CD3+CD4+FoxP3+CD127-

d. Increased frequency of $C D 3+C D 8+C D 28$ null (described as regulatory CD8 T cells) (data not shown) e. Decreased frequency of iNKT (data not shown)

\section{Cytokines immunological signature}

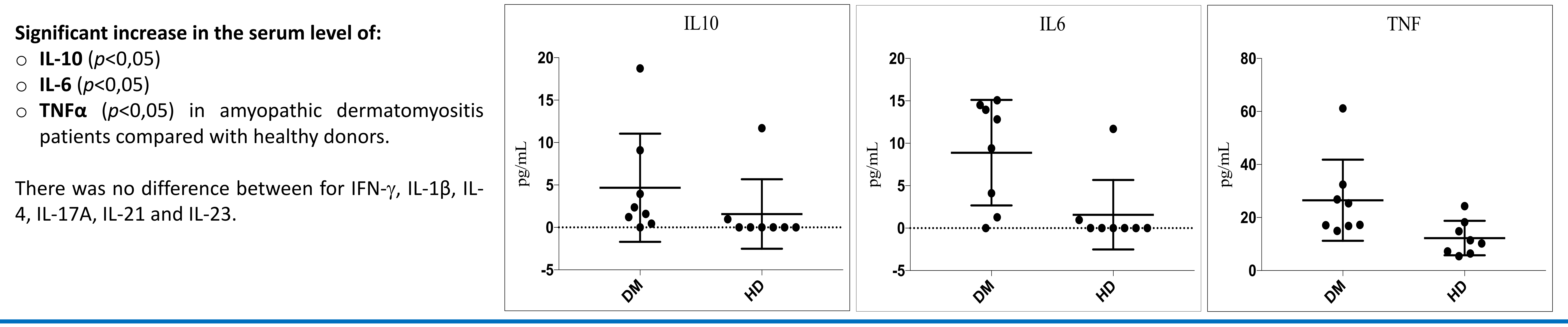

\section{Skin Transcriptome signature}

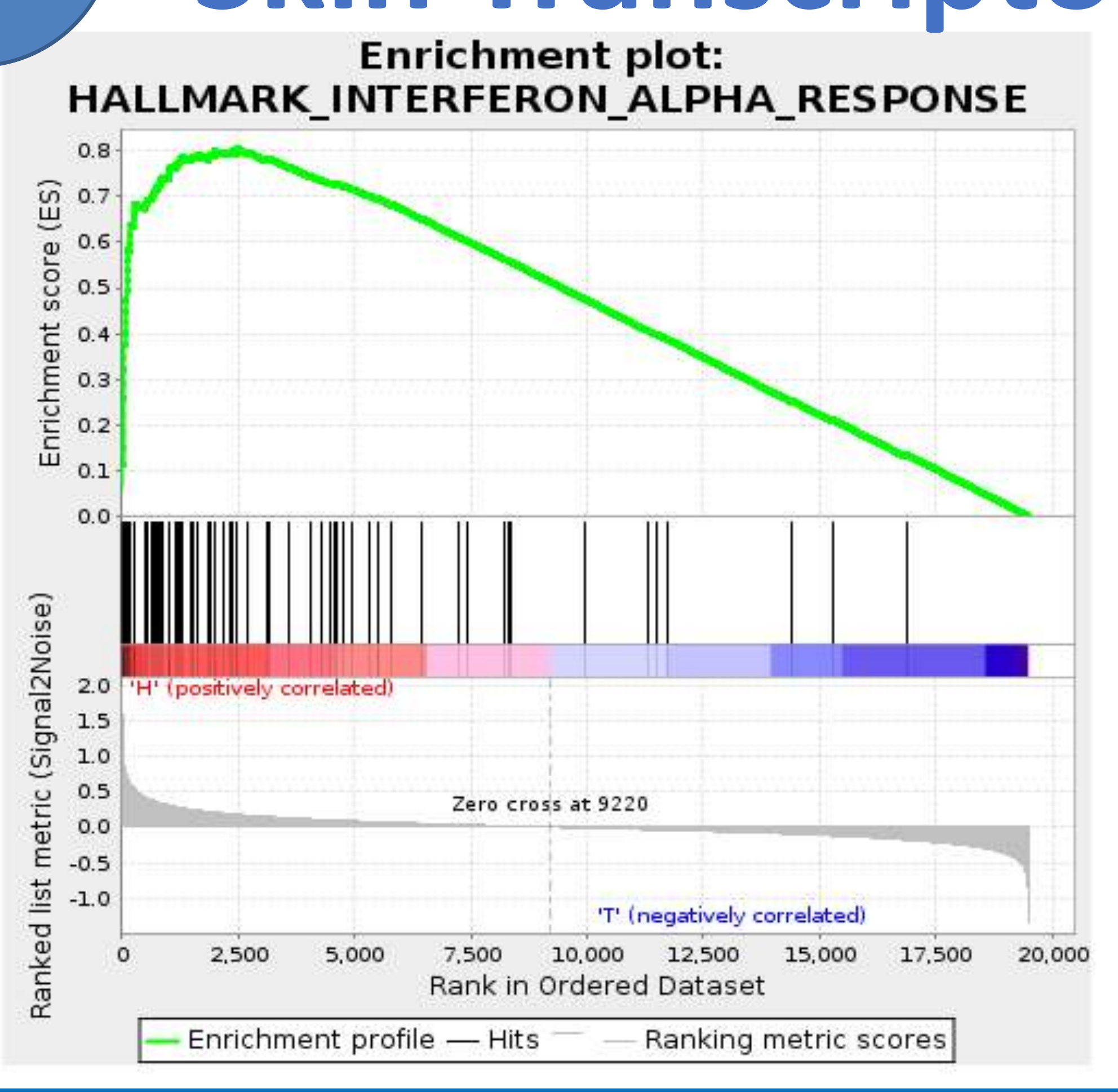

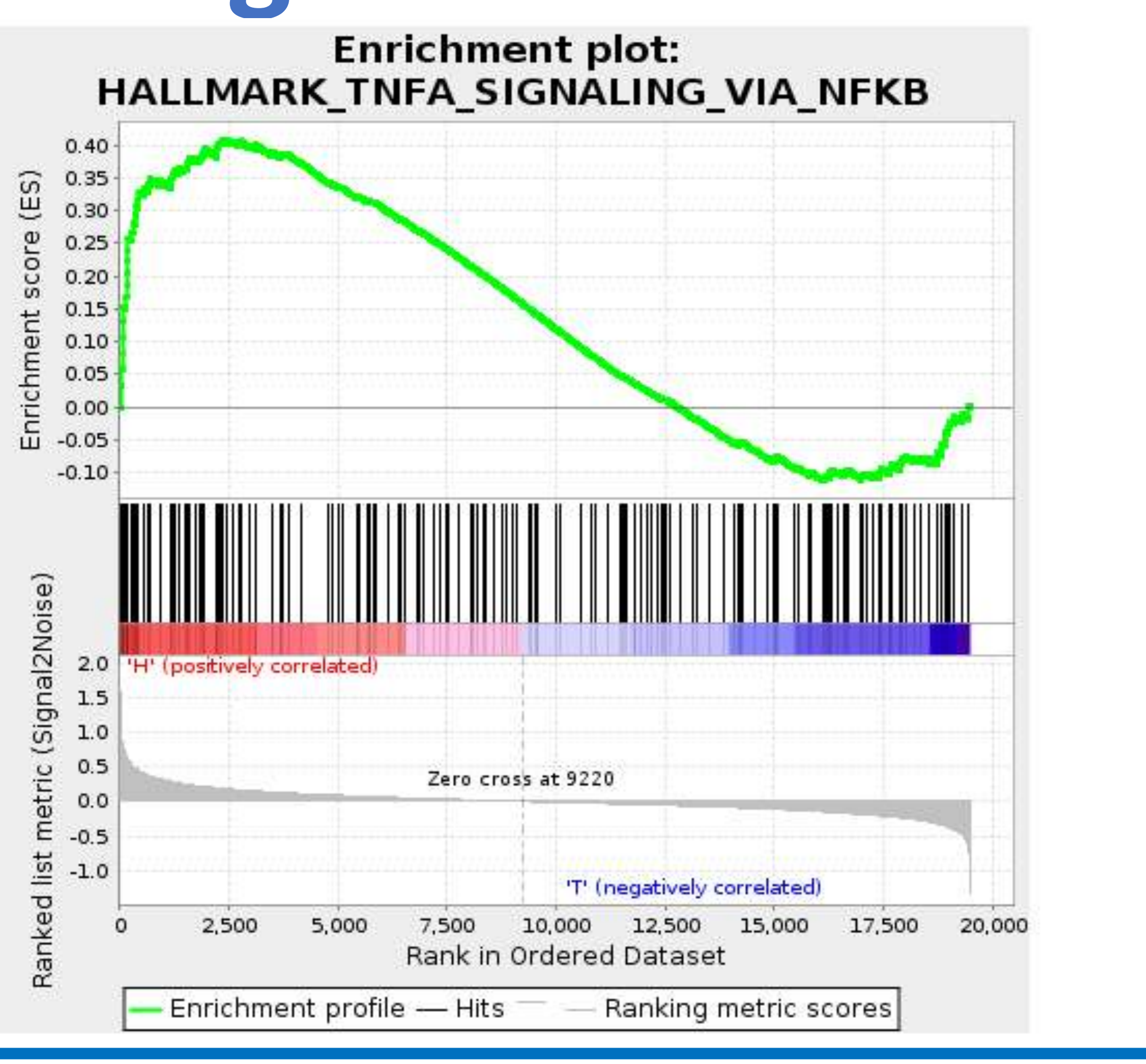

Pathway analyses revealed a significant enrichment in Interferon alpha response genes and cytotoxicity genes in aDM patients' skin compared to healthy patients' skin $(F D R=0,0)$

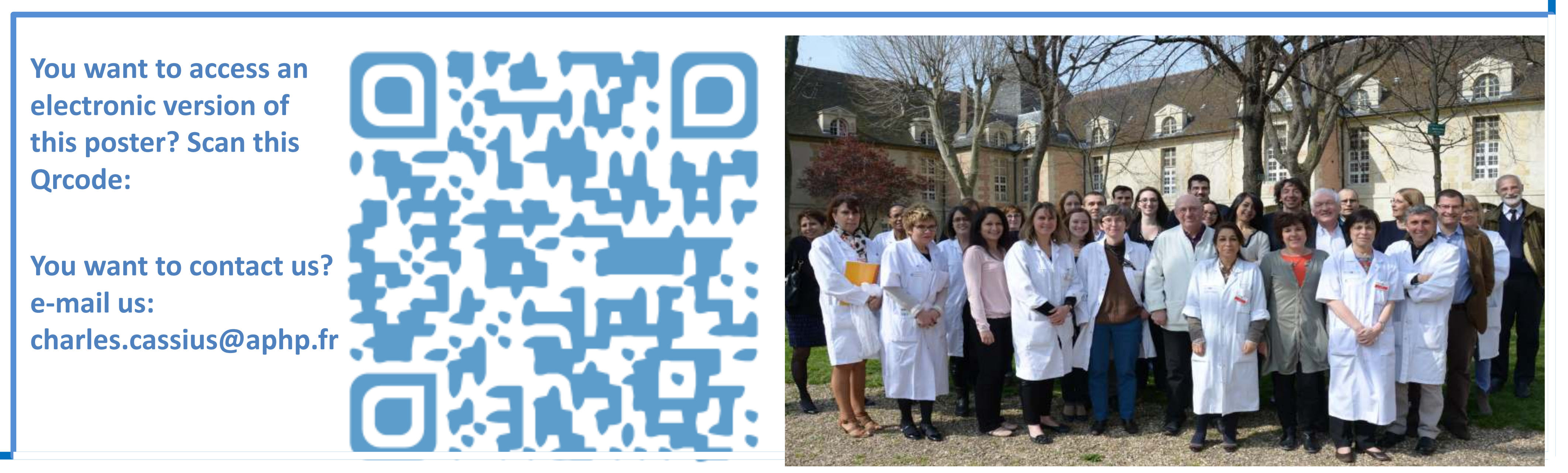

\section{Conclusion}

Our study evidences a systemic inflammation in aDM patients. We confirm some immunopathological hypotheses evoked in cDM. Further transcriptomic and cellular investigations will pave the way for the use of innovative systemic and topical targeted 